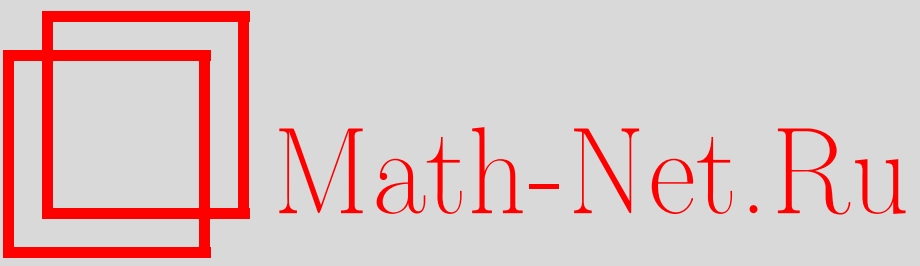

И. М. Бурлаков, Геометрия линейных алгебр, Итоги науки и техн. Сер. Соврем. мат. и ее прил. Темат. обз., 2020, том 182, 3-9

DOI: https://doi.org/10.36535/0233-6723-2020-182-3-9

Использование Общероссийского математического портала Math-Net.Ru подразумевает, что вы прочитали и согласны с пользовательским соглашением

http://www.mathnet.ru/rus/agreement

Параметры загрузки:

IP : 54.209 .52 .79

26 апреля 2023 г., 08:01:24 


\title{
ГЕОМЕТРИЯ ЛИНЕЙНЫХ АЛГЕБР
}

\author{
(c) 2020 г. $\quad$ И. М. БУРЛАКОВ
}

\begin{abstract}
АннотАция. В статье рассмотрены пространства, геометрия которых порождается однородной функцией степени $m \geqslant 2$, инвариантной относительно действия какой-либо подгруппы линейной группы данного пространства. Предложен общий способ и даны примеры реализации таких пространств на линейных алгебрах.
\end{abstract}

Ключевые слова: фундаментальная форма, группа движений, линейная алгебра, векторное расслоение.

\section{GEOMETRY OF LINEAR ALGEBRAS}

\author{
(c) 2020 I. M. BURLAKOV
}

\begin{abstract}
In this paper, we consider spaces whose geometry is generated by a homogeneous function of degree $m \geqslant 2$ invariant under the action of some subgroup of the linear group of the given space. A general method is proposed and examples of realization of such spaces on linear algebras are given.
\end{abstract}

Keywords and phrases: fundamental form, motion group, linear algebra, vector bundle.

AMS Subject Classification: 15A66, 15A69, 16S38

1. Возьмем $n$-мерное линейное пространство $L_{n}$ и зададим на нем фундаментальную форму

$$
\boldsymbol{g}_{m}(x)=g_{k_{1} k_{2} \ldots k_{m}} x^{k_{1}} x^{k_{2}} \ldots x^{k_{m}},
$$

где $\boldsymbol{x}=x^{i} \boldsymbol{e}_{i}$-произвольный вектор пространства $\boldsymbol{L}_{n}$, записанный в каком-либо базисе $\boldsymbol{e}_{1}, \boldsymbol{e}_{2}, \ldots, \boldsymbol{e}_{n}$. Тогда, чтобы посредством формы (1) на пространстве $\boldsymbol{L}_{n}$ «конструировать» те или иные геометрические величины, мы должны указать еще некоторую подгруппу $\boldsymbol{G}_{m} \subset \boldsymbol{G} \boldsymbol{L}(n)$, преобразования которой сохраняют фундаментальную форму.

В этом состоит подход Клейна к определению различных геометрий, который получил название «Эрлангенской программы» (см. [5]). В частности, если в качестве фундаментальной

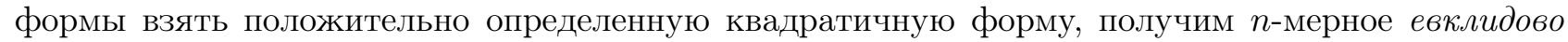
пространство. В случае же, когда для невырожденной квадратичной формы мы отказываемся

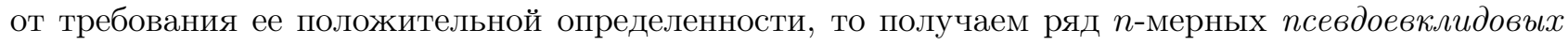
пространств различной сигнатуры. В связи с этим пространства с фундаментальными формами произвольной степени $m$ естественно называть почти евклидовыми пространствами (см. [1]).

В отличие от евклидовых и псевдоевклидовых пространств, геометрия пространств с фундаментальными формами, степень которых больше двух, изучена существенно меньше. Исследования таких пространств представлены в основном рассмотрением отдельных пространств с фиксированной фундаментальной формой. Примером может служить четырехмерное пространство Бервальда-Моора (см. [4]), фундаментальная форма которого имеет вид $\boldsymbol{g}_{4}(x)=x^{1} x^{2} x^{3} x^{4}$.

Вероятно, имеются две причины того, что геометрии пространств с фундаментальными формами степени выше 2 не привлекали особого внимания геометров. Во-первых, геометрия пространств с неквадратичной фундаментальной формой не находила приложений в естествознании 
(в отличие, скажем, от пространств со знакопеременной квадратичной формой, которые лежат в основе теории относительности). Во-вторых, трудно найти группы линейных преобразований, сохраняющих фундаментальную форму, степень которой будет больше двух. Если линейные преобразования, сохраняющие квадратичную форму, можно отыскать средствами теории матриц, то для поиска преобразований, сохраняющих форму степени выше двух, требуется гораздо более сложный аппарат тензорной алгебры или теории многомерных матриц.

2. Ситуация, однако, изменится, если появится простой способ, позволяющий отыскать группу линейных преобразований, сохраняющих какую-либо форму той или иной степени. Этот способ связан с линейными алгебрами и в общих чертах может быть описан следующим образом.

Пусть $\boldsymbol{K}_{n}$ - какая-либо унитальная алгебра, а $\boldsymbol{\Gamma}\left(\boldsymbol{K}_{n}\right)$ - группа обратимых элементов алгебры $\boldsymbol{K}_{n}$. Пусть $\boldsymbol{L}_{k} \subseteq \boldsymbol{K}_{n}$ - такое линейное подпространство, что для любых $x \in \boldsymbol{L}_{k}$ и любых элементов $a$ и $b$ некоторой подгруппы $\boldsymbol{G} \subset \boldsymbol{\Gamma}\left(\boldsymbol{K}_{n}\right)$ всегда $a \cdot x \cdot b \in \boldsymbol{L}_{k}$. Если найдется такая функция $\varphi: \boldsymbol{K}_{n} \rightarrow \boldsymbol{K}_{n}$, что $\varphi(x) \in \mathbb{R}$ и $\varphi(a \cdot x \cdot b)=\varphi(x)$ для любых $a, b \in \boldsymbol{G}$ и $x \in \boldsymbol{L}_{n}$, то на подпространстве $\boldsymbol{L}_{k} \subseteq \boldsymbol{K}_{n}$ возникает почти евклидова геометрия, фундаментальная форма которой получается ограничением функции $\varphi(x)$ на $\boldsymbol{L}_{k}$, т.е. $\boldsymbol{g}(x)=\left.\varphi(x)\right|_{\boldsymbol{L}_{k}}$, а движениями в этой почти евклидовой геометрии будут преобразования подпространства $\boldsymbol{L}_{k}$, порождаемые линейными алгебраическими функциями $x^{\prime}=a \cdot x \cdot b$, где $a, b \in \boldsymbol{G}$.

Классическим примером являются алгебры гиперкомплексных чисел Клифборда $A_{n}^{r}$ или алгебра альтернионов (см. [7]). Базис линейного пространства этой алгебры состоит из единицы, элементов $\boldsymbol{e}_{1}, \boldsymbol{e}_{2}, \ldots, \boldsymbol{e}_{n}$ и всевозможных произведений $\boldsymbol{e}_{i_{1} i_{2} \ldots i_{q}}=\boldsymbol{e}_{i_{1}} \boldsymbol{e}_{i_{2}} \ldots \boldsymbol{e}_{i_{q}}$, где $i_{1}<i_{2}<\ldots<i_{q}$, т.е. для любого $a \in A_{n}^{r}$

$$
a=a_{0}+\sum_{i=1}^{n} a_{i} \boldsymbol{e}_{i}+\sum_{i<h} a_{i h} \boldsymbol{e}_{i h}+\sum_{i<h<l} a_{i h l} \boldsymbol{e}_{i h l}+\ldots+a_{12 \ldots n} \boldsymbol{e}_{12 \ldots n},
$$

где $a_{i_{1} i_{2} \ldots i_{q}} \in \mathbb{R}$. При этом умножение гиперкомплексных чисел Клиффорда задается следующими структурными тождествами:

$$
\boldsymbol{e}_{h} \boldsymbol{e}_{l}=\boldsymbol{e}_{l} \boldsymbol{e}_{h}, h \neq l, \quad \boldsymbol{e}_{1}^{2}=\cdots=\boldsymbol{e}_{r}^{2}=1, \quad \boldsymbol{e}_{r+1}^{2}=\cdots=\boldsymbol{e}_{n}^{2}=-1 .
$$

Заметим еще, что в $A_{n}^{r}$ определена инволюция $\overline{\boldsymbol{e}_{i_{1} i_{2} \ldots i_{h-1} i_{h}}}=\boldsymbol{e}_{i_{h} i_{h-1} \ldots i_{2} i_{1}}$, так что имеют место соотношения

$$
\overline{a+b}=\bar{a}+\bar{b}, \quad \overline{a \cdot b}=\bar{b} \cdot \bar{a}, \quad \overline{\bar{a}}=a \quad \forall a, b \in A_{n}^{r} .
$$

Подпространство $\boldsymbol{L}_{n} \subset A_{n}^{r}$, натянутое на элементы $\boldsymbol{e}_{1}, \boldsymbol{e}_{2}, \ldots, \boldsymbol{e}_{n}$, называется подстилающим пространством алгебры альтернионов. Возьмем всевозможные элементы $q_{h} \in \boldsymbol{L}_{n}$, для которых $q_{h}^{2}= \pm 1$, и обозначим через $\operatorname{Pin} A_{n}^{r}$ мультипликативную группу, элементами которой будут всевозможные произведения $q_{1} \cdot q_{2} \cdot \ldots \cdot q_{h}$ таких элементов. Тогда функция $\varphi(x)=x \cdot \bar{x}$ задает на подстилающем пространстве квадратичную форму, которая определяет на $\boldsymbol{L}_{n}$ евклидову или псевдоевклидову геометрию, а линейные функции

$$
x^{\prime}=(-1)^{h} q_{h} \cdots q_{2} \cdot q_{1} \cdot x \cdot \overline{q_{h} \cdots q_{2} \cdot q_{1}}
$$

задают собственные и несобственные вращения пространства $\boldsymbol{L}_{n}$, так как функция $x^{\prime}=-q \cdot x \cdot q$ определяет зеркальное отражение от гиперплоскости, ортогональной вектору $q$, а любое вращение можно представить как композицию четного числа зеркальных симметрий (см. [7]).

3. Пример почти евклидовых геометрий с неквадратичной фундаментальной формой дают циклические алгебры $\mathbb{R}_{m}(e)$, базис которых состоит из единицы и степеней $e, e^{2}, e^{3}, \ldots, e^{m-1}$ образующего элемента $e$, так что для произвольного элемента $x \in \mathbb{R}_{m}(e)$ имеем

$$
x=x_{0}+x_{1} e+x_{2} e^{2}+\cdots+x_{m-1} e^{m-1}, \quad x_{k} \in \mathbb{R} .
$$


Произведение в алгебре $\mathbb{R}_{m}(e)$ определяется тождеством $e^{m}=1$. Можно показать, что величина

$$
\Delta_{m}(x)=\left|\begin{array}{ccccc}
x_{0} & x_{m-1} & x_{m-2} & \ldots & x_{1} \\
x_{1} & x_{0} & x_{m-1} & \ldots & x_{2} \\
x_{2} & x_{1} & x_{0} & \ldots & x_{3} \\
\ldots & \ldots & \ldots & \ldots & \ldots \\
x_{m-1} & x_{m-2} & x_{m-3} & \cdots & x_{0}
\end{array}\right|,
$$

называемая детерминантом элемента $x \in \mathbb{R}_{m}(e)$, обладает свойством мультипликативности, т.е.

$$
\Delta_{m}(x \cdot y)=\Delta_{m}(x) \Delta_{m}(y) .
$$

Это свойство позволяет ввести на линейном пространстве алгебры $\mathbb{R}_{m}(e)$ структуру почти евклидова пространства с фундаментальной формой $\boldsymbol{g}_{m}(x)=\Delta_{m}(x)$, где движениями будут преобразования, порождаемые функциями $x^{\prime}=a \cdot x$, при условии, что $\Delta_{m}(a)=1$. Такая геометрия называется ииклической, а движения в циклических пространствах естественно называть циклическими вращениями (см. [2]).

4. Геометрия циклических пространств (как пример почти евклидовых геометрий с неквадратичной фундаментальной формой) имеет широкое обобщение, в основе которого лежит одно утверждение, которое ниже будет сформулировано как теорема. Предварительно требуется распространить на элементы любых алгебр $\boldsymbol{K}_{n}$ конструкцию детерминанта, которая выше была введена для циклических чисел.

Пусть $a, b \in \boldsymbol{K}_{n}-$ фиксированные элементы; тогда линейное алгебраическое уравнение $a \cdot x=b$ в некотором базисе $\boldsymbol{e}_{k}$ алгебры $\boldsymbol{K}_{n}$ будет эквивалентно системе линейных скалярных уравнений

$$
a^{k} \gamma_{k h}^{r} x^{h}=b^{r},
$$

где $\gamma_{k h}^{r}-$ структурные константы, т.е.

$$
\boldsymbol{e}_{k} \cdot \boldsymbol{e}_{h}=\gamma_{k h}^{r} \boldsymbol{e}_{r}
$$

Левым детерминантом $\Delta_{L}(a)$ элемента $a \in \boldsymbol{K}_{n}$ называется определитель системы (3), т.е.

$$
\Delta_{L}(a)=\operatorname{det}\left(a^{k} \gamma_{k h}^{r}\right) \text {. }
$$

Аналогично определяется правый детерминант

$$
\Delta_{R}(a)=\operatorname{det}\left(a^{h} \gamma_{k h}^{r}\right)
$$

элемента $a \in \boldsymbol{K}_{n}$ : он является определителем системы линейных уравнений

$$
a^{h} \gamma_{k h}^{r} x^{k}=b^{r}
$$

эквивалентной линейному алгебраическому уравнению $x \cdot a=b$.

Теорема 1 (о детерминанте). Левый (правый) детерминант элементов ассоциативных алгебр есть мультипликативная функиия.

Доказательство. Доказательство этой теоремы сразу следует из тождества ассоциативности $(a$. $b) \cdot x=a \cdot(b \cdot x)$, записанного в координатах:

$$
a^{k} b^{h} \gamma_{k h}^{r} \gamma_{r q}^{m} x^{q}=a^{k} \gamma_{k r}^{m} b^{h} \gamma_{h q}^{r} x^{q},
$$

откуда для левых детерминантов имеем

$$
\Delta_{L}(a \cdot b)=\operatorname{det}\left(\left(a^{k} b^{h} \gamma_{k h}^{r}\right) \gamma_{r q}^{m}\right)=\operatorname{det}\left(\left(a^{k} \gamma_{k r}^{m}\right)\left(b^{h} \gamma_{h q}^{r}\right)\right)=\Delta_{L}(a) \Delta_{L}(b) .
$$

Мультипликативность $\Delta_{R}$ доказывается аналогично.

Таким образом, каждая ассоциативная алгебра на своем линейном пространстве порождает некоторую почти евклидову геометрию, в которой роль фундаментальной формы играет левый (или правый) детерминант текущего элемента $x \in \boldsymbol{K}_{n}$, а движениями будут линейные преобразования пространства алгебры, порождаемые линейными алгебраическими функциями вида 
$x^{\prime}=a \cdot x \cdot b$, где $\Delta_{L}(a)=\Delta_{L}(b)=1$ (или $\Delta_{R}(a)=\Delta_{R}(b)=1$ ). Такую геометрию естественно называть детерминантной (см. [1]).

Заметим еще, что детерминантную геометрию можно определить и на неассоциативных алгебрах, если только детерминант элементов этих алгебр будет обладать свойством мультипликативности. Примером может служить алгебра октав.

5. Пример с алгебрами альтернионов (из п. 2) также допускает широкое обобщение. Для этого введем в рассмотрение алгебры $\boldsymbol{B}_{n}^{m}$, обобщающие алгебры комплексных альтернионов $C_{n}(\mathbb{C})$ так, что для любого вектора $x$ некоторого комплексного линейного пространства $\boldsymbol{L}_{n} \subset \boldsymbol{B}_{n}^{m}$ будет справедливо тождество

$$
x^{m} \equiv\left(x_{1} \boldsymbol{e}_{1}+x_{2} \boldsymbol{e}_{2}+\ldots+x_{n} \boldsymbol{e}_{n}\right)^{m}=x_{1}^{m}+x_{2}^{m}+\ldots+x_{n}^{m},
$$

где $\boldsymbol{e}_{1}, \boldsymbol{e}_{2}, \ldots, \boldsymbol{e}_{n}$ - базис пространства $\boldsymbol{L}_{n}$.

Линейным базисом алгебры $\boldsymbol{B}_{n}^{m}$ будут всевозможные произведения

$$
\boldsymbol{e}_{\beta_{1} \beta_{2} \ldots \beta_{n}}=\boldsymbol{e}_{1}^{b_{1}} \cdot \boldsymbol{e}_{2}^{b_{2}} \cdot \ldots \cdot \boldsymbol{e}_{n}^{b_{n}},
$$

где $\beta_{k}$-набор из $b_{k}$ индексов $k$, при этом $0 \leqslant b_{k}<m$. Произведение элементов алгебры $\boldsymbol{B}_{n}^{m}$ определяются структурными тождествами $\boldsymbol{e}_{k}^{m}=1$ и

$$
\boldsymbol{e}_{k} \cdot \boldsymbol{e}_{h}=\left\{\begin{array}{ll}
\alpha_{m} \boldsymbol{e}_{h} \cdot \boldsymbol{e}_{k} & \text { для } k>h, \\
\bar{\alpha}_{m} \boldsymbol{e}_{h} \cdot \boldsymbol{e}_{k} & \text { для } k<h
\end{array},\right.
$$

где $\alpha_{m}=\cos (2 \pi / m)+i \sin (2 \pi / m)$. Легко видеть, что $\boldsymbol{B}_{n}^{m}=C_{n}(\mathbb{C})$, т.е. алгебры $\boldsymbol{B}_{n}^{m}$ на самом деле являются обобщением алгебр комплексных альтернионов, для которых $\alpha_{2}=-1$, и для векторов подстилающего пространства алгебры $C_{n}(\mathbb{C})$ тождество $(5)$ имеет вид

$$
x^{2} \equiv\left(x_{1} \boldsymbol{e}_{1}+x_{2} \boldsymbol{e}_{2}+\ldots+x_{n} \boldsymbol{e}_{n}\right)^{2}=x_{1}^{2}+x_{2}^{2}+\ldots+x_{n}^{2} .
$$

Алгебры $\boldsymbol{B}_{n}^{m}$ были введены в рассмотрение М. П. Бурлаковым в 1970-х гг. для описания пространств симметрий кварков различных поколений и получили название элементальных алгебр $m$-й степени (см. [1-3]).

На линейных пространствах элементальных алгебр естественно возникают различные почти евклидовы геометрии. Остановимся на рассмотрении одного примера таких пространств, который реализуется на пространстве алгебры $\boldsymbol{B}_{2}^{3}$. Заметим, что текущий элемент $x$ этой алгебры можно записать в виде

$$
x=x_{00}+x_{10} \boldsymbol{e}_{1}+x_{01} \boldsymbol{e}_{01}+x_{20} \boldsymbol{e}_{20}+x_{11} \boldsymbol{e}_{11}+x_{02} \boldsymbol{e}_{02}+x_{21} \boldsymbol{e}_{21}+x_{12} \boldsymbol{e}_{12}+x_{22} \boldsymbol{e}_{22} .
$$

При этом можно показать, что кубическая форма

$$
\begin{gathered}
\varphi_{3}(x)=x_{00}^{3}+x_{10}^{3}+x_{20}^{3}-3 x_{00} x_{10} x_{20}+ \\
+x_{01}^{3}+x_{11}^{3}+x_{21}^{3}-3 x_{01} x_{11} x_{21}+x_{02}^{3}+x_{12}^{3}+x_{22}^{3}-3 x_{02} x_{12} x_{22}- \\
-3\left(x_{00} x_{01} x_{02}+\alpha_{3}^{2} x_{10} x_{21} x_{02}+\alpha_{3} x_{20} x_{11} x_{02}+\alpha_{3}^{2} x_{00} x_{11} x_{22}+\alpha_{3} x_{10} x_{01} x_{22}+\right. \\
\left.\quad+x_{20} x_{21} x_{22}+\alpha_{3} x_{00} x_{21} x_{12}+x_{10} x_{11} x_{12}+\alpha_{3}^{2} x_{20} x_{01} x_{12}\right) \in \mathbb{C},
\end{gathered}
$$

где $\alpha_{3}=(-1+\sqrt{3} i) / 2$, обладает свойством мультипликативности, т.е. $\varphi_{3}(x \cdot y)=\varphi_{3}(x) \varphi_{3}(y)$ для любых $x, y \in \boldsymbol{B}_{2}^{3}$ (см. [1]).

Поэтому форму $\boldsymbol{g}_{6}(x)=\varphi_{3}(x) \overline{\varphi_{3}(x)}$ можно принять за фундаментальную форму 18 -мерного (вещественного) почти евклидова пространства, движениями которого будут преобразования, задаваемые линейными алгебраическими функциями $x^{\prime}=a \cdot x \cdot b$, при условии, что $\varphi_{3}(a)=$ $\exp (\gamma i)$ и $\varphi_{3}(b)=\exp (\delta i)$, где $\gamma, \delta \in \mathbb{R}$ - какие-либо фиксированные числа. Конечно, возникает вопрос: существуют ли такие элементы алгебры $\boldsymbol{B}_{2}^{3}$, от которых значение формы $\varphi_{3}(x)$ было бы экспонентой чисто мнимой величины? Ответ на этот вопрос положительный. В частности, можно показать, что если $c(t)=\exp \left(t \boldsymbol{e}_{\sigma}\right)$, где $\sigma$-любой мультииндекс базисных элементов алгебры $\boldsymbol{B}_{2}^{3}$, 
то $\varphi_{3}(c(t))=1$. Таким образом, элементы $c(t)$, где $t \in \mathbb{C}$, определяют однопараметрические подгруппы группы движений почти евклидова пространства алгебры $\boldsymbol{B}_{2}^{3}$.

Заметим еще, что аналогичные геометрические структуры можно определять и на произвольных элементальных алгебрах (см. [1]).

6. Какие геометрические величины, аналогичные основным евклидовым величинам, можно ввести в почти евклидовых пространствах посредством фундаментальной формы (1)?

Прежде всего, это будет некоторая инвариантная мера векторов линейного пространства $\boldsymbol{L}_{n}$, на котором реализована структура почти евклидовой геометрии. Эта инвариантная мера для произвольного вектора $x \in \boldsymbol{L}_{n}$ определяется формулой

$$
\rho(x)=\sqrt[m]{\boldsymbol{g}_{m}(x)} \equiv \sqrt[m]{g_{k_{1} k_{2} \ldots k_{m}} x^{k_{1}} x^{k_{2}} \ldots x^{k_{m}}} .
$$

Очевидно, что если вектор $x \in \boldsymbol{L}_{n}$ умножить на положительное вещественное число $k$, то и величина $\rho(x)$ умножится на это же число, т.е. $\rho(k x)=k \rho(x)$. Поэтому величину $\rho(x)$ естественно называть почти евклидовой длиной вектора $x$.

Однако почти евклидова длина вектора $x \in \boldsymbol{L}_{n}$, определенная по формуле (8), будет, вообще говоря, комплексным числом, поэтому наряду с величиной $\rho(x)$ целесообразно рассматривать еще и положительную вещественную величину

$$
|x|=\sqrt[m]{\left|\boldsymbol{g}_{m}(x)\right|} \equiv \sqrt[m]{\left|g_{k_{1} k_{2} \ldots k_{m}} x^{k_{1}} x^{k_{2}} \ldots x^{k_{m}}\right|} .
$$

которую можно назвать почти евклидовым модулем вектора $x \in \boldsymbol{L}_{n}$.

Располагая аналогом длины в почти евклидовых пространствах, мы можем ввести конструкцию, заменяющую в этих пространствах сферу. Множество векторов $x \in \boldsymbol{L}_{n}$, удовлетворяющих уравнению

$$
g_{k_{1} k_{2} \ldots k_{m}} x^{k_{1}} x^{k_{2}} \ldots x^{k_{m}}=r^{m}
$$

будем называть сфероидом с радиусом $r$. Если при этом $r=0$, то сфероид называется изотропным. В аффинном пространстве, где векторы можно считать приложенными к некоторой точке, уравнение (10) представляет собой гиперповерхность, которую естественно также называть noчти евклидовым сфероидом.

$\mathrm{K}$ примеру, в циклической алгебре $\mathbb{R}_{2}(e)$ фундаментальная форма дается детерминантом

$$
\Delta_{2}(x)=\left|\begin{array}{ll}
x_{0} & x_{1} \\
x_{1} & x_{0}
\end{array}\right|=x_{0}^{2}-x_{1}^{2}
$$

т.е. в алгебре $\mathbb{R}_{2}(e)$ реализуется псевдоевклидова планиметрия, в которой неизотропные сфероиды будут гиперболами $x_{0}^{2}-x_{1}^{2}=r^{2}$, а изотропный сфероид будет парой пересекающихся прямых $x_{0}= \pm x_{1}$.

В качестве еще одного примера возьмем циклическую геометрию третьего порядка, для которой фундаментальная форма имеет вид

$$
\Delta_{3}(x)=\left|\begin{array}{lll}
x_{0} & x_{2} & x_{1} \\
x_{1} & x_{0} & x_{2} \\
x_{2} & x_{1} & x_{0}
\end{array}\right|=x_{0}^{3}+x_{1}^{3}+x_{2}^{3}-3 x_{0} x_{1} x_{2} .
$$

Можно показать, что изотропный сфероид в $\mathbb{R}_{3}(e)$ образован плоскостью с уравнением $x_{0}+x_{1}+$ $x_{2}=0$ и прямой с уравнением $x_{0}=x_{1}=x_{2}$. Если в трехмерном пространстве алгебры $\mathbb{R}_{3}(e)$ перейти к изотропному базису, векторы которого лежат на изотропном сфероиде, то уравнение неизотропного сфероида примет вид

$$
x=2 r^{3} / 3 \sqrt{3}\left(y^{2}+z^{2}\right) .
$$

Отсюда видно, что поверхность, изображающая сфероид циклической геометрии третьего порядка в трехмерном евклидовом (или аффинном) пространстве, получается евклидовым вращением квадратичной гиперболы с уравнением $x=2 r^{3} / 3 \sqrt{3} y^{2}$ вокруг оси $O x$. При этом прямая $x_{0}=x_{1}=x_{2}$ является асимптотой этой поверхности, а плоскость $x_{0}+x_{1}+x_{2}=0$ - асимптотической плоскостью. 
7. В евклидовой геометрии важнейшей геометрической величиной наряду с длиной векторов является угол между двумя векторами. На евклидовой плоскости, реализованной в алгебре комплексных чисел, величину угла между парой векторов можно определить следующим образом.

Любое комплексное число $z=x+i y$, представляющее вектор на евклидовой плоскости, можно однозначно записать в экспоненциальной форме:

$$
z=r(\cos \psi+i \sin \psi)=r \exp (\psi i), \quad r \in[0 ;+\infty), \quad \psi \in[0 ; 2 \pi) .
$$

Тогда величина угла между векторами $z_{1}=r_{1} \exp \left(\psi_{1} i\right)$ и $z_{2}=r_{2} \exp \left(\psi_{2} i\right)$ будет равна разности $\psi=\psi_{2}-\psi_{1}$. При этом такая разность инвариантна относительно любых поворотов, так как любые повороты даются линейными функциями указанного вида: $z^{\prime}=\exp (\lambda i) z$.

Заметим еще, что без утраты смысла в качестве угла между векторами $z_{1}$ и $z_{2}$ на евклидовой плоскости можно брать чисто мнимое число $\left(\psi_{2}-\psi_{1}\right) i$, что будет даже более естественно, так как мы не можем складывать величины углов и длины векторов.

Такой подход к определению углов между парами векторов можно распространить и на другие почти евклидовы геометрии, реализованные на линейных пространствах некоторых алгебр.

Например, на псевдоевклидовой плоскости, представленной циклической алгеброй $\mathbb{R}_{2}(e)$, любой элемент $x=x_{0}+x_{1} e \in \mathbb{R}_{2}(e)$, у которого $x_{0}>0$ и $\left|x_{1}\right|<x_{0}$, можно записать в так называемой гиперболической форме:

$$
x=r(\operatorname{ch} \xi+e \operatorname{sh} \xi)=r \exp (\xi e), \quad r \in(0 ;+\infty), \quad \xi \in(-\infty ;+\infty) .
$$

Тогда величина псевдоевклидова угла между векторами $x_{1}=r_{1} \exp \left(\xi_{1} e\right)$ и $x_{2}=r_{2} \exp \left(\xi_{2} e\right)$ будет равна разности $\xi=\xi_{2}-\xi_{1}$. Эта разность инвариантна относительно любых псевдоевклидовых поворотов, так как они задаются линейными функциями вида $x^{\prime}=\exp (\lambda e) \cdot x$. Здесь снова в качестве угловой меры целесообразнее вместо разности $\xi=\xi_{2}-\xi_{1}$ брать $\left(\xi_{2}-\xi_{1}\right) e$, чтобы различать линейные и угловые величины.

Возьмем еще почти евклидову геометрию с кубической фундаментальной формой

$$
\boldsymbol{g}_{3}(x)=x_{0}^{3}+x_{1}^{3}+x_{2}^{3}-3 x_{0} x_{1} x_{2},
$$

которая реализуется на циклической алгебре $\mathbb{R}_{3}(e)$. Любой элемент $x=x_{0}+x_{1} e+x_{2} e^{2}$ этой алгебры, у которого

$$
x_{0}+x_{1}+x_{2}>0, \quad\left(x_{0}-x_{1}\right)^{2}+\left(x_{0}-x_{1}\right)^{2}+\left(x_{0}-x_{1}\right)^{2} \neq 0,
$$

можно представить в экспоненииальном виде:

$$
x=r \exp \left(\mu e+\eta e^{2}\right), \quad r \in(0 ;+\infty), \quad \mu, \eta \in(-\infty ;+\infty) .
$$

Тогда в качестве меры почти евклидова угла между векторами $x_{1}, x_{2} \in \mathbb{R}_{3}(e)$ можно взять разность

$$
\theta=\left(\mu_{2}-\mu_{1}\right) e+\left(\eta_{2}-\eta_{1}\right) e^{2} \in \mathbb{R}_{3}(e),
$$

которая инвариантна относительно собственных движений в почти евклидовом пространстве $\mathbb{R}_{3}(e)$ : можно показать, что собственные движения в $\mathbb{R}_{3}(e)$ даются линейными функциями

$$
x^{\prime}=x \cdot \exp \left(\mu e+\eta e^{2}\right) .
$$

Из приведенных примеров видно, что угол в почти евклидовых пространствах - аналог евклидова угла, - можно ввести в пространствах, где существуют однопараметрические группы с аддитивным параметром, соединяющие различные пары векторов.

Завершая краткий обзор почти евклидовых геометрий, следует еще сказать, что в этих геометриях можно ввести и меры площади и объемов. Мы не обсуждаем в данной работе конструкцию таких величин в силу ограниченности объема статьи. 
8. Геометрии почти евклидовых пространств локально можно перенести на гладкие многообразия, подобно тому как евклидова геометрия локально переносится на гладкие многообразия, порождая на них риманову геометрию. Для этого на гладком многообразии $\boldsymbol{M}_{n}$ надо задать фундаментальную форму

$$
\boldsymbol{g}_{m}[X](v)=g[X]_{k_{1} k_{2} \ldots k_{m}} v^{k_{1}} v^{k_{2}} \ldots v^{k_{m}},
$$

зависящую от точки многообразия $X \in \boldsymbol{M}_{n}$ и принимающую значения на векторах $v$ касательных пространств $\boldsymbol{T}_{\boldsymbol{X}}$. Таким образом, на каждом касательном пространстве возникает почти евклидова геометрия. При этом на $\boldsymbol{M}_{n}$ можно определять геометрические величины, заимствуя их с касательных пространств. Например, полагая

$$
d s^{m}=g[X]_{k_{1} k_{2} \ldots k_{m}} d x^{k_{1}} d x^{k_{2}} \ldots d x^{k_{m}}
$$

мы можем определить почти евклидову длину линии $X(t)$, соединяющей две точки $X(a)$ и $X(b)$, вычислив для этого интеграл от формы (12) вдоль заданной линии. Определенную таким образом геометрию на гладком многообразии с заданной на нем формой (11) естественно называть почти римановым пространством.

Геометрия почти римановых пространств определяется некоторой формой степени $m \geqslant 2$. Как и в случае почти евклидовых пространств, фундаментальную форму на гладком многообразии целесообразно задавать при помощи алгебраической структуры, представляющей собой некоторое обобщение векторного расслоения.

Типовым слоем векторного расслоения $\left(\boldsymbol{V}_{m}, \boldsymbol{M}_{n}, p\right)$ является векторное пространство $\boldsymbol{V}_{m}$, так что в каждом слое $\boldsymbol{V}_{m}(X) \equiv p^{-1}(X)$, где $X \in \boldsymbol{M}_{n}$, имеется структура векторного пространства, не зависящая от выбора координатного гомеоморфизма (см. [6]). Аналогично определяется алгебраическое расслоение $\left(\boldsymbol{K}_{m}, \boldsymbol{M}_{n}, p\right)$, в котором типовым слоем будет линейная алгебра $\boldsymbol{K}_{m}$, так что в каждом слое $\boldsymbol{K}_{m}(X) \equiv p^{-1}(X)$, где $X \in \boldsymbol{M}_{n}$, имеется структура линейной алгебры, не зависящая от выбора координатного гомеоморфизма. При этом множество сечений алгебраического расслоения автоматически наделяется структурой (вообе говоря, бесконечномерной) линейной алгебры.

Если алгебра $\boldsymbol{K}_{m}$ на некотором своем линейном подпространстве $\boldsymbol{L}_{n}$ определяет почти евклидову геометрию, как это было описано выше, то, отождествляя $\boldsymbol{L}_{n}(X)$ с касательным пространством $\boldsymbol{T}_{X}$, мы тем самым введем на многообразии $\boldsymbol{M}_{n}$ структуру почти риманова пространства, в котором фундаментальная форма определяет геометрические величины для сечений расслоения $\left(\boldsymbol{K}_{m}, \boldsymbol{M}_{n}, p\right)$.

\section{СПИСОК ЛИТЕРАТУРЫ}

1. Бурлаков И. М., Бурлаков М. П. Геометрические структуры линейных алгебр. - LAP LAMBERT, 2016.

2. Бурлаков М. П. Гамильтоновы алгебры. - М.: Граф Пресс, 2006.

3. Бурлаков М. П. Алгебраические методы в геометрической физике. - Грозный, 1985.

4. Гарасъко Г. И. Начала финслеровой геометрии для физиков. - М.: ТЕТРУ, 2009.

5. Клейн Ф. Элементарная математика с точки зрения высшей. - М.: Наука, 1987.

6. Мищенко А. С. Векторные расслоения и их применения. - М.: Наука, 1984.

7. Розенфельд Б. А. Неевклидовы геометрии. - М.: ГИТТЛ, 1955.

Бурлаков Игорь Михайлович

Тверской государственный университет

E-mail: don. burlakoff@yandex.ru 